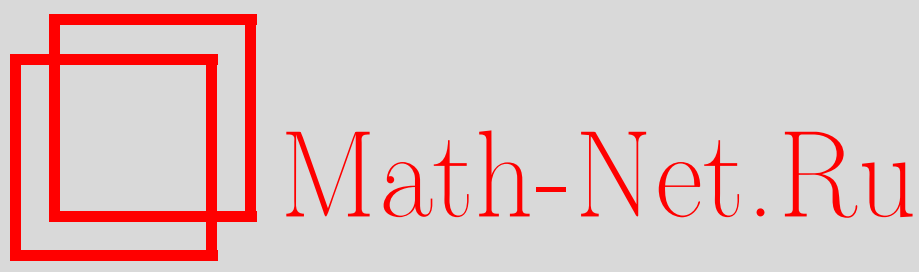

И. Х. Сабитов, Алгоритмическое решение проблемы изометрической реализации двумерных многогранных метрик, Изв. РАН. Сер. матем., 2002, том 66, выпуск 2, 159-172

DOI: https://doi.org/10.4213/im382

Использование Общероссийского математического портала Math-Net.Ru подразумевает, что вы прочитали и согласны с пользовательским соглашением

http://www . mathnet.ru/rus/agreement

Параметры загрузки:

IP: 54.162 .127 .20

26 апреля 2023 г., 03:03:49 
УДК 513.7

\author{
И. Х. Сабитов
}

\title{
Алгоритмическое решение проблемы изометрической реализации двумерных многогранных метрик
}

\begin{abstract}
Для многогранников в общем положении и с данным комбинаторным строением указывается алгоритм нахождения всех их метрических характеристик: объемы, двугранные углы и диагонали - через длины их ребер и тем самым устанавливается возможность развития нового направления геометрических исследований, которое, по аналогии с известным термином "решение треугольников", можно назвать "решением многогранников".

Библиографиия: 17 наименований.
\end{abstract}

1. В работах автора [6]-[8], [15] была доказана теорема, представляющая собой обобщение формулы Герона для площади треугольника на объемы ориентируемых многогранников. Именно, пусть $P$ - ориентируемый многогранник с комбинаторным строением $K$. Это означает, что для симплициального комплекса $K$, триангулируюшего некоторое ориентируемое многообразие $M$, дано его непрерывное отображение $P: K \rightarrow \mathbb{R}^{3}$, линейное на каждом симплексе комплекса $K$, в предположении, что $K$ задан как некоторое множество евклидовых треугольников и их сторон и вершин. Геометрический образ $P(K)$ многогранника в $\mathbb{R}^{3}$ может иметь очень сложное строение, с какими-то вырождениями, самопересечениями и т.д. Поэтому понятие оббема для таких многогранников требует специального уточнения, состоящего в том, что объем $V=\operatorname{vol}(P)$ понимается в обобшенном смысле как сумма ориентированных объемов тетраэдров, натянутых на некоторую общую их вершину $O$ и на согласованно ориентированные грани многогранника, а ориентация на $P(K)$ перенесена отображением $P: K \rightarrow \mathbb{R}^{3}$ из произвольно выбранной ориентации многообразия $M$. Таким образом определенный объем не зависит от выбора точки $O$, и для вложенных многогранников он совпадает с их обычным ориентированным объемом.

Обозначим через $(l)=\left(l_{1}^{2}, \ldots, l_{e}^{2}\right)$ совокупность квадратов длин ребер многогранника, пронумерованных в некотором порядке, где $e$ - число ребер. Тогда упомянутое выше обобшение формулы Герона можно сформулировать в виде следующей теоремы.

ТЕОРема 1. Для любого многогранника $P$ с треугольными гранями существует полиномиальное уравнение

$$
Q(V, l)=V^{2 N}+a_{1}(l) V^{2 N-2}+\cdots+a_{N-1}(l) V^{2}+a_{N}(l)=0
$$

такое, что обобщенный объем $V$ многогранника $P$ является корнем этого уравнения, в котором коэффициенты $a_{i}$ являются многочленами от $(l)=$

Работа выполнена при частичной финансовой поддержке РФФИ (грант № 99-01-00867) и ИНТАС-РФФИ (грант № 97-01-71010).

(C) И.х. САвитов, 2002 
$\left(l_{1}^{2}, \ldots, l_{e}^{2}\right)$ с некоторыми рациональными числовыми коэффичиентами, зависящими только от комбинаторного строения $K$ многогранника ${ }^{1}$.

Таким образом получено, что объемы всех многогранников, изометричных в классическом смысле многограннику $P$ (т.е. имеющих одинаковое с $P$ комбинаторное строение с гранями, конгруәнтнылми соответствующим граням из $P$, и тем самым не допускающих граней с так называемыми "плавающими" ребрами; см. о них, например, в [16]), тоже являются корнями того же уравнения (1), и, следовательно, получается, что объем многогранника является функцией (вообще говоря, многозначной) его метрики.

Для последующих результатов важно знать алгебраический смысл уравнения (1). Пусть $\left(x_{3 i+1}, x_{3 i+2}, x_{3 i+3}\right), 0 \leqslant i \leqslant n-1,-$ координаты всех $n$ вершин многогранника, и пусть точка $O$ выбрана в начале координат. Тогда ориентированный объем каждого тетраэдра вычисляется через смешанное произведение векторов, идущих из начала координат в вершины граней многогранника, и поэтому обобшенный объем $V$ многогранника будет выражаться через координаты вершин как некоторый однородный многочлен степени 3. Далее, квадраты длин ребер тоже выражаются через координаты вершин как некоторые однородные многочлены степени 2. Это мы будем записывать как

$$
V=V(x), \quad l=l(x)
$$

имея в виду, что $x$ обозначает совокупность всех координат всех вершин, занумерованных в некотором произвольном, но фиксированном порядке. То, что объемы всех изометричных многогранников являются корнями одного и того же полиномиального уравнения (1), выражается следуюшим алгебраическим свойством многочлена $Q(V, l)$ : если мы подставим в $Q$ значения $V$ и $l$ из $(2)$ как многочленов от $x$, то получим тождественный нуль, т.е.

$$
Q(V(x), l(x)) \equiv 0 \quad \text { относительно всех } x_{1}, \ldots, x_{3 n} .
$$

Если для некоторого многочлена $F(V, l)$ будет выполнено тождество

$$
F(V(x), l(x)) \equiv 0
$$

то назовем $F$ многочленом для оббема многогранника $P$.

Оказалось, что возможность вычисления объема многогранника по его внутренней геометрии позволяет найти алгоритмическое решение практически всех основных задач метрической теории многогранников, по крайней мере, тех, которые находятся в общем положении ${ }^{2}$. В настоящей работе приводятся некоторые примеры применения теоремы 1 к этим задачам. Ранее, в кратком изложении, эти результаты были опубликованы в [9] (см. также [10]).

\footnotetext{
${ }^{1}$ Строго говоря, в том многочлене $Q$, который построен для доказательства теоремы, степень $2 N$ многочлена и его коэффициенты зависят еще и от некоторого произвола в выборе способа построения многочлена (это допускается исключительно в рамках, определяемых свойствами комбинаторного строения многогранника, а не его метрикой или пространственной конфигурацией); однако для гомеоморфных сфере многогранников существует так называемый канонический многочлен с наименьшей степенью $2 N$, который определяется $е д и н-$ ственным образом по комбинаторному строению $K$ многогранника (см. [3]).

2 Это значит, что координаты вершин $\left(x_{1}, \ldots, z_{n}\right)$ многогранника с данным комбинаторным строением не удовлетворяют никакому алгебраическому уравнению.
} 
2. Начнем с проблемы изометрической реализации в $\mathbb{R}^{3}$ данной полиэдральной метрики. В нашей постановке эта проблема формулируется так: задается некоторое множество $K$ евклидовых треугольников с таким правилом отождествления их сторон и вершин, при котором пересечение любой пары треугольников либо пусто, либо состоит из одной общей вершины или из одной общей стороны, так что после такого отождествления будет получено некоторое компактное ориентируемое многообразие, для которого $K$ является его триангуляцией; ищется многогранник $P: K \rightarrow \mathbb{R}^{3}$ такой, что треугольники из $K$ являются его гранями (в том смысле, что образ $P(F)$ каждой грани $F$ из $K$ является треугольником в $\mathbb{R}^{3}$, конгруэнтным $F$ ). Таким образом, отображение $P: K \rightarrow \mathbb{R}^{3}$ должно дать изометрическое погружение (но лучше говорить об “изометрической реализаиии”, так как в топологическом смысле погружения может и не быть) метрического симплициального комплекса $K$ в $\mathbb{R}^{3}$ с сохранением двумерных симплексов как граней. В случае существования такой реализации комплекс $K$ будет представлять собой натуральную развертку многогранника $P(K)$. В такой постановке задачи об изометрическом погружении до недавнего времени не было известно никакого - ни положительного, ни отрицательного - результата ${ }^{3}$. Наша теорема позволяет получить следующее очевидное необходимое условие изометрической реализуемости многогранной метрики: для того чтобы данная метрическим симплициальным комплексом $K$ многогранная метрика была изометрически погружаема (вложима) в $\mathbb{R}^{3}$, необходимо, чтобы многочлен $Q(V)$, построенный по комбинаторному строению и метрике $K$, имел хотя бы один неотрицательный (положительный) корень $V^{2}$.

Проблема нахождения какого-либо достаточного условия изометрической реализуемости данной многогранной метрики остается пока открытой, но можно указать алгоритм проверки такой возможности. Для этого покажем, что для многогранников в общем положении сушествует алгоритм, позволяющий установить конечность множества возможных значений двугранных углов между гранями во всех парах граней с общим ребром и одновременно дающий способ вычисления значений этих углов. Очевидно, знание величины двугранного угла между двумя гранями с общим ребром равносильно знанию длины диагонали, соединяющей те две вершины граней, которые не инцидентны их общему ребру. Назовем такие диагонали мальм.м.

Покажем, что верна следующая

ТЕОРЕма 2. Для каждой малой диагонали есть полиномиальное уравнение, коэффициенты которого зависят только от метрики и комбинаторного строения многогранника и для многогранников в общем положении они не все равны нулю.

Для доказательства теоремы нужна следующая

ЛЕМма 1. Пусть $l_{k}$ - длина произвольным образом выбранного ребра в многогранниках данного комбинаторного строения $K$. Тогда не может быть, чтобы между длинами ребер этих многогранников существовала за-

\footnotetext{
${ }^{3} \mathrm{~B}$ известных работах [1], [4] о существовании изометрического погружения полиэдральных метрик не предполагается и, вообще говоря, не полуцается, что данная в виде $K$ развертка искомого многогранника является натуральной.
} 
висимость вида 4

$$
L\left(\hat{l}_{k}, l\right)=A_{0}\left(\hat{l}_{k}\right) l_{k}^{S}+\cdots+A_{S-1}\left(\hat{l}_{k}\right) l_{k}+A_{S}\left(\hat{l}_{k}\right)=0
$$

такая, что $A_{0}^{2}\left(\hat{l}_{k}\right)+\cdots+A_{S-1}^{2}\left(\hat{l}_{k}\right) \neq 0$ nрu $l_{1}^{2}+\cdots+l_{k-1}^{2}+l_{k+1}^{2}+\cdots+l_{e}^{2} \neq 0$, где е - число ребер, а запись $\hat{l}_{k}$ означает, что в аргументе участвуют все длины $l_{1}, \ldots, l_{e}$, кроме $l_{k}$.

ДокАЗАТЕЛЬСтво. Пусть в $K$ конщевые вершины $k$-го ребра имеют номера $i$ и $j$, априлегаюшие к этому ребру грани $F$ и $G$ имеют вершины $i, j, p$ и $i, j, q$. Построим в $\mathbb{R}^{3}$ многогранник $P(K)$ по следуюшему отображению $P: K \rightarrow \mathbb{R}^{3}:$ вершинам $i, j, p$ и $q$ сопоставим соответственно точки $M_{i}\left(0, y_{i}, z_{i}\right), M_{j}\left(0, y_{j}, z_{j}\right), M_{p}(a, 0,0)$ и $M_{q}(-a, 0,0), a \neq 0$, а все остальные вершины отобразим в некоторые точки на оси $O x$. Теперь будем вращать точки $M_{i}$ и $M_{j}$ вокруг оси $O x$, оставляя все остальные вершины на месте. В процессе такой деформации получим новые многогранники $P_{t}$ с тем же комбинаторным строением $K$. При этом длины всех ребер, кроме $k$-го, не изменяются, и поэтому наличие уравнения (4) с указанным в условиях леммы свойством означало бы, что при данных значениях длин $l_{1}, \ldots, l_{k-1}, l_{k+1}, \ldots, l_{e}$, не равных нулю одновременно, длина $l_{k}$ могла бы иметь только некоторое конечное число значений, что невозможно ввиду непрерывного изменения $l_{k}$ в процессе вращения многогранника. Лемма доказана.

ЗАмЕчАниЕ 1. Справедливость леммы для гомеоморфных сфере многогранников является следствием жесткости строго выпуклых многогранников и возможности построения для каждой триангулящии сферы изоморфного ей выпуклого многогранника (см. [3]).

ЗАмечАниЕ 2. Полезно заметить, что приведенное доказательство леммы дает возможность также получить следующее утверждение.

УТВЕРЖДЕНИЕ 1. Пусть $l_{1}, \ldots, l_{e}-$ длины ребер в многогранниках одного и того же комбинаторного строения $K$. Тогда в любой многочлен для обгема әтих многогранников каждая длина $l_{k}$ как переменная входит по крайней мере в один из коэффичиентов многочлена.

ДоКАЗАТЕЛЬСТво. Обобщенный объем построенного при доказательстве леммы многогранника равен $V=\frac{1}{6} 2 a\left(y_{j} z_{i}-y_{i} z_{j}\right.$ ) (объемы тетраэдров с основаниями по всем граням, кроме $F$ и $G$, равны нулю). При врашении точек $M_{i}$ и $M_{j}$ вокруг оси $O x$, с сохранением остальных вершин на месте, будем получать новые многогранники $P_{t}$ с разными объемами. Но если бы в многочлене для объема переменная $l_{k}$ - длина $k$-го ребра - не участвовала, то ввиду постоянства всех длин, кроме $l_{k}$, объемы многогранников $P_{t}$ тоже имели бы постоянное значение, что и даст требуемое противоречие.

ДоКАЗАТЕЛЬСТво ТЕОРЕМЫ 2. Пусть $\langle C D\rangle$ - малая диагональ в многограннике $P$ для двугранного угла между гранями $\langle A B C\rangle$ и $\langle A B D\rangle$ с общим ребром $\langle A B\rangle$. Выбросим эти две грани и заклеим образовавшуюся дыру двумя новыми гранями $\langle A D C\rangle$ и $\langle B D C\rangle$; получим новый многогранник $P_{1}$, для которого малая

\footnotetext{
${ }^{4}$ Тот факт, что уравнение (4) выполняется для длин ребер именно многогранников, а не для длин одномерных симплексов комплекса $K$, означает, что подстановка в (4) значений $l=l(x)$ из $(2)$ обращает это уравнение в тождество $L\left(\hat{l}_{k}(x), l(x)\right) \equiv 0$.
} 


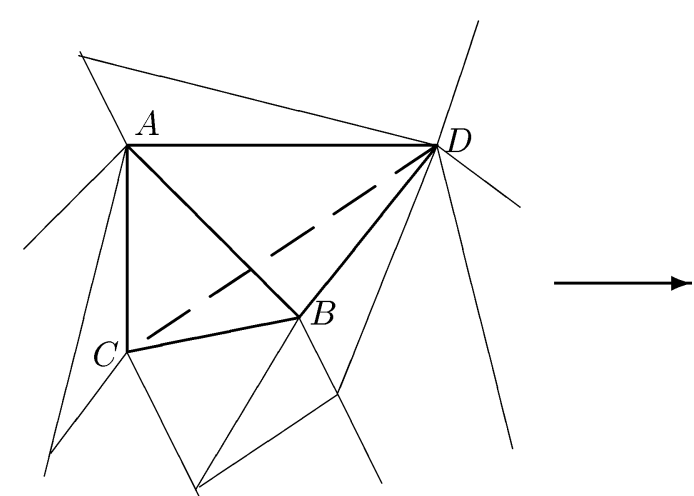

Многогранник $P$

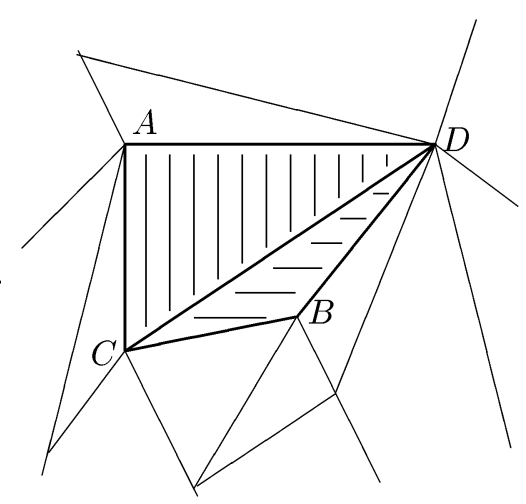

Многогранник $P_{1}$

Рис. 1

диагональ $\langle C D\rangle$ многогранника $P$ является одним из ребер (см. рис. 1). Подобно тому, как объем $V=\operatorname{vol}(P)$ является корнем уравнения (1), объем $V_{1}=\operatorname{vol}\left(P_{1}\right)$ является корнем некоторого уравнения вида ${ }^{5}$

$$
Q_{1}=V_{1}^{2 N}+a_{1}(\hat{l}, d) V_{1}^{2 N-2}+\cdots+a_{N}(\hat{l}, d)=0,
$$

в котором коэффициенты $a_{i}, 1 \leqslant i \leqslant N$, зависят от длин ребер многогранника $P$ (но не от длины ребра $\langle A B\rangle$, что отражено в обозначении $\hat{l}$ ) и длины $d$ диагонали $\langle C D\rangle$. Объемы $V=\operatorname{vol}(P)$ и $V_{1}=\operatorname{vol}\left(P_{1}\right)$ отличаются на геометрический объем $V_{T} \geqslant 0$ тетраэдра $\langle A B C D\rangle$ :

$$
V_{1}=V+\epsilon V_{T}, \quad \epsilon= \pm 1 .
$$

В общем случае неизвестно, что требуется: прибавить или вычесть объем тетраэдра, поэтому между $V$ и $V_{1}$ необходимо найти соотношение, справедливое при любой ориентации и при любом взаимном расположении рассматриваемых многогранников. Для этого исключаем $\epsilon$ и получаем уравнение

$$
V_{1}^{4}-2\left(V^{2}+V_{T}^{2}\right) V_{1}^{2}+\left(V^{2}-V_{T}^{2}\right)^{2}=0 .
$$

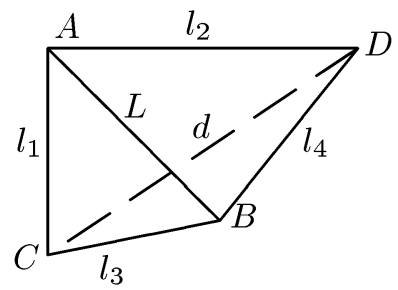

Рис. 2

\footnotetext{
${ }^{5}$ Степень многочлена $Q_{1}$ в общем случае, конечно, другая, чем в $(1)$, скажем $2 N_{1}$, но чтобы избежать обилия индексов, мы оставили то же обозначение $2 N$.
} 
Если рассматривать (5) и (6) как уравнения относительно общей переменной $v=V_{1}^{2}$, то получим, что их результант $R$ будет равен нулю:

$$
R=\left|\begin{array}{ccccccc}
1 & a_{1} & a_{2} & a_{3} & \ldots & a_{N} & 0 \\
0 & 1 & a_{1} & a_{2} & \ldots & a_{N-1} & a_{N} \\
1 & (*) & \left(V^{2}-V_{T}^{2}\right)^{2} & 0 & \ldots & 0 & 0 \\
\vdots & \vdots & \vdots & \ddots & \vdots & \vdots & \vdots \\
\vdots & \vdots & \vdots & \vdots & \ddots & \vdots & \vdots \\
0 & 0 & 0 & \ldots & \ldots & \left(V^{2}-V_{T}^{2}\right)^{2} & 0 \\
0 & 0 & 0 & \cdots & \cdots & (*) & \left(V^{2}-V_{T}^{2}\right)^{2}
\end{array}\right|=0
$$

где для краткости записи использовано обозначение $(*)=-2\left(V^{2}+V_{T}^{2}\right)$. Объем тетраэдра (см. рис. 2) вычисляется по известной формуле

$$
\begin{aligned}
144 V_{T}^{2}= & L^{2} d^{2}\left(l_{1}^{2}+l_{3}^{2}+l_{2}^{2}+l_{4}^{2}-L^{2}-d^{2}\right)+l_{1}^{2} l_{4}^{2}\left(L^{2}+d^{2}+l_{2}^{2}+l_{3}^{2}-l_{1}^{2}-l_{4}^{2}\right) \\
& +l_{3}^{2} l_{2}^{2}\left(L^{2}+d^{2}+l_{1}^{2}+l_{4}^{2}-l_{3}^{2}-l_{2}^{2}\right)-L^{2}\left(l_{1}^{2} l_{2}^{2}+l_{3}^{2} l_{4}^{2}\right)-d^{2}\left(l_{1}^{2} l_{3}^{2}+l_{2}^{2} l_{4}^{2}\right)
\end{aligned}
$$

(обозначения длин понятны из рисунка). Раскрывая определитель (7), собирая слагаемые по степеням $d$ и учитывая формулу (8), получим относительно $d$ некоторый многочлен вида

$$
A_{0}(\hat{l}, L, V) d^{2 K}+A_{1}(\hat{l}, L, V) d^{2 K-2}+\cdots+A_{K}(\hat{l}, L, V)=0 .
$$

Уравнения (5) и (6) верны для всех многогранников с одним и тем же данным комбинаторным строением, т.е. они выполнены тождественно относительно $x=$ $\left(x_{1}, \ldots, x_{3 n}\right)$, когда вместо длин ребер, диагоналей и объемов подставлены их значения (2) через координаты вершин многогранников. Это значит, что если в коэффициенты уравнений (5) и (6) относительно $v=V_{1}^{2}$ подставлены значения диагонали $d$, объема $V$ и длин всех ребер многогранника $P$ как функций от $x$, то они всегда имеют обшее решение $v(x)=V_{1}^{2}(x)$, поэтому

$$
R(x)=R(\hat{l}(x), L(x), d(x), V(x)) \equiv 0 .
$$

Наша задача - показать, что если в (9) оставить $d$ как переменную, то тождественного равенства нулю относительно переменных $x, d$ не будет. Это равносильно тому, что при подстановке в коэффициенты $A_{i}$ уравнения (9) вместо длин ребер и объема их выражений (2) через координаты вершин мы получим хотя бы один коэффициент, который не окажется равным тождественно нулю. Для этого исследуем распределение в (9) степеней переменной $L$. По теореме о весе общий $j$-й член (моном) результанта (7) имеет вид

$$
c_{j} a_{1}^{\mu_{1}^{(j)}} \ldots a_{N}^{\mu_{N}^{(j)}}(-2)^{\nu_{0}^{(j)}}\left(V^{2}+V_{T}^{2}\right)^{\nu_{1}^{(j)}}\left(V^{2}-V_{T}^{2}\right)^{2 \nu_{2}^{(j)}},
$$

где $c_{j}$ - некоторое отличное от нуля целое число, а неотрицательные целые числа $\mu_{0}^{(j)}, \mu_{1}^{(j)}, \ldots, \nu_{2}^{(j)}$ удовлетворяют соотношениям

$$
\begin{gathered}
\mu_{0}^{(j)}+\mu_{1}^{(j)}+\cdots+\mu_{N}^{(j)}=2, \quad \nu_{0}^{(j)}+\nu_{1}^{(j)}+\nu_{2}^{(j)}=N, \\
\mu_{1}^{(j)}+2 \mu_{2}^{(j)}+\cdots+N \mu_{N}^{(j)}+\nu_{1}^{(j)}+2 \mu_{2}^{(j)}=2 N .
\end{gathered}
$$


Найдем, какова наибольшая степень, с которой переменная $L$ входит в результант. В коэффициентах $a_{i}$ переменной $L$ нет вовсе, поэтому в каждом мономе (11) она встречается только в множителях $\left(V^{2}+V_{T}^{2}\right)^{\nu_{1}^{(j)}}\left(V^{2}-V_{T}^{2}\right)^{2 \nu_{2}^{(j)}}$, значит, с учетом (8) и (12), в наивысшей степени она входит в результант в мономе при ${ }^{6} \mu_{0}=2$, $\mu_{1}=\cdots=\mu_{N}=0, \nu_{0}=\nu_{1}=0, \mu_{2}=N$. Значит, этот единственный моном имеет вид $c L^{8 N} d^{4 N}$, где постоянная $c=\frac{1}{144^{2 N}}$. Выпишем коэффициент $A_{2 K-4 N}(\hat{l}, L, V)$ при $d^{4 N}$. В нем обязательно есть слагаемое $c L^{8 N}$, которому не с чем уничтожиться, так как во всех остальных слагаемых переменная $L$ имеет меньшую степень, следовательно, $A_{2 K-4 N}(\hat{l}, L, V) \neq 0$ тождественно как функция выписанных аргументов. Тем самым мы, во-первых, доказали, что $R(\hat{l}, L, V, d)$ как многочлен от $d$ не равен нулю тождественно, во-вторых, получили оценку его степени: $2 K \geqslant 4 N$.

Представим $A_{2 K-4 N}(\hat{l}, L, V)$ как многочлен относительно $V$, и пусть он равен нулю как функция от $x$. Тогда получим уравнение вида

$$
B_{0}(\hat{l}, L) V^{2 M}+\cdots+B_{M-1}(\hat{l}, L) V^{2}+B_{M}(\hat{l}, L)=0
$$

где степени всех коэффициентов $B_{i}, 0 \leqslant i \leqslant M-1$, относительно $L$ меньше $8 N$, а

$$
B_{M}(\hat{l}, L)=c L^{8 N}+B_{M}^{(0)}(\hat{l}, L)
$$

причем степень $B_{M}^{(0)}$ относительно $L$ меньше $8 N$.

Выпишем теперь коэффициент $A_{K}(\hat{l}, L, V)$ в $(9)$ при $d^{0}$, в него, в частности, войдет моном $V^{4 N}$ из $R$, которьй получается из обшего члена (11) результанта при $\mu_{0}=2, \mu_{1}=\cdots=\mu_{N}=0, \nu_{0}=\nu_{1}=0, \mu_{2}=N$, причем во всех остальных мономах степень $V$ меньше, чем $4 N$. Мы предположили, что все коэффициенты в (9) при ненулевых степенях $d$ обращаются тождественно в нуль относительно $x$; тождественно равен нулю относительно $x$ и сам результант. Следовательно, коэффищиент $A_{K}$ тоже должен равняться нулю. Тогда имеем

$$
V^{4 N}+C_{1}(\hat{l}, L) V^{4 N-2}+\cdots+C_{2 N}(\hat{l}, L)=0 .
$$

Уравнения (13) и (15) являются однородными по размерности, т.е. все слагаемые в них имеют одну и ту же размерность ${ }^{7}$, равную $\left[\right.$ ед. длины] ${ }^{8 N}$ для (13) и $[\text { ед. длины }]^{12 N}$ для (15). Следовательно, в коэффициенты $B_{i}(\hat{l}, L), 0 \leqslant i \leqslant M$, переменная $L$ может входить только в степенях, не превышаюших $8 N-6 M+6 i$, а в коэффициентах $C_{s}(\hat{l}, L), 1 \leqslant s \leqslant 2 N$, степень $L$ не больше, чем $6 s$ (и притом меньше, чем $8 N$ ). На самом деле степени $L$ в коэффициентах $C_{s}$ строго меньше, чем $6 s$. Действительно, из структуры общего члена (11) результанта и формулы (8) для $V_{T}$ видно, что переменная $L$ при нулевой степени $d$ встречается только вместе с другими длинами как сомножителями, поэтому в многочленах $C_{s}$ вклад степеней $L$ в размерность $6 s$ каждого монома в $C_{s}$ будет меньше, чем $6 s$, а недостаюшая часть размерности обеспечится степенями других сомножителей этого монома. Обозначим степени $L$ в $B_{i}$ через $\beta_{i}$, а в $C_{s}-$ через $\gamma_{s}$. Тогда

$$
\beta_{i} \leqslant 8 N-6 M+6 i, \quad 0 \leqslant i \leqslant M, \quad \gamma_{s}<6 s, \quad 1 \leqslant s \leqslant 2 N .
$$

\footnotetext{
${ }^{6}$ Действительно, если $\nu_{1}+2 \nu_{2}=2 N$, то равенство $\nu_{0}+\nu_{1}+\nu_{2}=N$ возможно только при $\nu_{0}=\nu_{1}=0, \nu_{2}=N$.

7 Это общее свойство всех встречающихся в наших рассмотрениях многочленов.
} 
Исключим теперь из уравнений (13) и (15) общую переменную $v=V^{2}$. Получим результант

$$
R_{1}=\left|\begin{array}{ccccccc}
1 & C_{1} & \ldots & \ldots & \ldots & 0 & 0 \\
0 & 1 & \ldots & \ldots & \ldots & 0 & 0 \\
\vdots & \vdots & \ddots & \vdots & \vdots & \vdots & 0 \\
0 & 0 & \ldots & 1 & \ldots & C_{2 N-1} & C_{2 N} \\
B_{0} & B_{1} & \ldots & & B_{M} & \ldots & 0 \\
\vdots & \vdots & \vdots & \vdots & \vdots & \ddots & \vdots \\
0 & 0 & \ldots & \ldots & \ldots & B_{M-1} & B_{M}
\end{array}\right|=0 .
$$

Общий член результанта имеет вид

$$
c_{j} C_{1}^{\mu_{1}^{(j)}} \ldots C_{2 N}^{\mu_{2 N}^{(j)}} B_{0}^{\nu_{0}^{(j)}} B_{1}^{\nu_{1}^{(j)}} \ldots B_{M}^{\nu_{M}^{(j)}}
$$

где показатели степеней связаны равенствами

$$
\begin{gathered}
\mu_{0}^{(j)}+\mu_{1}^{(j)}+\cdots+\mu_{2 N}^{(j)}=M, \quad \nu_{0}^{(j)}+\nu_{1}^{(j)}+\cdots+\nu_{M}^{(j)}=2 N, \\
\mu_{1}^{(j)}+2 \mu_{2}^{(j)}+\cdots+2 N \mu_{2 N}^{(j)}+\nu_{1}^{(j)}+2 \nu_{2}^{(j)}+\cdots+M \nu_{M}^{(j)}=2 M N .
\end{gathered}
$$

С учетом вышеприведенных гранищ для степеней $L$ в коэффициентах $C_{s}$ и $B_{i}$ оценим степень, с какой может входить $L$ в мономы результанта $R_{1}$. Возьмем в $(17)$ в каждом сомножителе наивысшую допустимую степень $L$. Тогда с учетом (16) и (19) получим

$$
\begin{aligned}
\gamma_{1} \mu_{1}^{(j)} & +\gamma_{2} \mu_{2}^{(j)}+\cdots+\gamma_{2 N} \mu_{2 N}^{(j)}+\beta_{0} \nu_{0}^{(j)}+\beta_{1} \nu_{1}^{(j)}+\cdots+\beta_{M} \nu_{M}^{(j)} \\
\leqslant & 6\left(\mu_{1}^{(j)}+2 \mu_{2}^{(j)}+\cdots+2 N \mu_{2 N}^{(j)}\right)+(8 N-6 M)\left(\nu_{0}^{(j)}+\cdots+\nu_{M}^{(j)}\right) \\
& +6\left(\nu_{1}^{(j)}+2 \nu_{2}^{(j)}+\cdots+M \mu_{M}^{(j)}\right)=16 N^{2},
\end{aligned}
$$

причем равенство бывает только в том случае, если множители при $\gamma_{s}$ равны нулю (так как в (16) для $\gamma_{s}$ имеем строгие неравенства), т.е. при $\mu_{1}^{(j)}=\mu_{2}^{(j)}=\cdots$ $\cdots=\mu_{2 N}^{(j)}=0$. А тогда $\nu_{0}^{(j)}=\nu_{1}^{(j)}=\cdots=\nu_{M-1}^{(j)}=0, \nu_{M}^{(j)}=2 N$. Следовательно, наивысшая степень $L$ в результанте (17) бывает в единственном мономе $c^{2 N} L^{16 N^{2}}$. Тем самым получаем, что тождество $R_{1}(\hat{l}(x), L(x)) \equiv 0$ сводится к тому, что равенство

$$
c_{0} L^{16 N^{2}}+c_{1}(\hat{l}) L^{16 N^{2}-2}+\cdots+c_{8 N^{2}}(\hat{l})=0, \quad c_{0}=c^{2 N},
$$

преврашается в тождество относительно $x$, т.е. в многогранниках такого комбинаторного строения $K$, как у $P$, одно ребро определяется значениями других ребер, что по лемме 1 невозможно. Тем самым не может быть, чтобы многочлен (9) относительно диагонали $d$ был нулевым. Теорема 2 доказана.

Из этой теоремы немедленно получаем такие следствия.

СЛЕДСТВИЕ 1. Для многогранников в общем положении с данным комбинаторныцм строением и с данными значениями длин ребер их малье диагонали могут принимать только конечное число значений, определяемых как корни уравнений вида (9), в каждом из которых вместо $V^{2}$ подставлены поочередно возможные значения квадрата обгема многогранника, получаемые как неотрицательные корни уравнения (1) относительно $V^{2}$. 
СЛЕДСТВИЕ 2. Для того чтобы многогранник был изгибаемым, необходимо, чтобы хотя бы для одной малой диагонали в соответствующем уравнении (9) все коэффичиенты $A_{i}(l, V), 0 \leqslant i \leqslant K$, были равны нулю при значениях длин ребер и обгема этого многогранника.

СлеДСТВИЕ 3. Почти все многогранники в $\mathbb{R}^{3}$ являются неизгибаемыми.

Утверждение следствия 3 в случае многогранников рода $g=0$ доказано в [12], для рода $g \geqslant 1$ оно известно только в препринтных публикациях: [13] и [17] для случая $g=1$, а для общего случая - в [14], с очень сложным доказательством (правда, для любой размерности).

ЗАмЕчАниЕ 3 . При вычислении малых диагоналей можно и не обрашаться к значениям объема многогранника. Для этого достаточно исключить переменную $V$ из уравнений (1) и (9) и получить полиномиальное уравнение степени $2 K N$ относительно $d^{2}$ с коэффициентами, зависящими только от длин ребер. Но при этом возрастет число ненужных вычислений, так как при использовании объемов мы учитываем только неотрицательные значения корней $V^{2}$ уравнения (1), и поэтому число значений $d^{2}$ получается, вообще говоря, меньше, чем $2 K N$.

Из следствия 1 получается следуюший алгоритм построения многогранников с их данной натуральной разверткой: находятся все неотрицательные корни $V^{2}$ уравнения (1), затем они по очереди подставляются в каждое из уравнений (9) (в обшем случае их число равно $e$ - числу ребер многогранника), находятся неотрицательные корни $d^{2}$ всех этих уравнений и тем самым составляется список всех возможных значений длин всех малых диагоналей, затем по этому списку составляется новый список, представляющий собой все варианты длин совокупности малых диагоналей для каждого искомого многогранника, т.е. для каждой малой диагонали берется одно из возможных значений ее длины ${ }^{8}$. Если все диагонали многогранника являются "малыми", то это делается достаточно легко. Такова комбинаторная схема, например, октаэдров, и для них этот алгоритм реализован аспирантом МГУ С. Н. Михалевым на компьютерах с использованием системы Maple. Приведем пример работы его программы, которая выполнена по методу, описанному в замечании 3 .

Пусть дан изоморфный октаэдру метрический симплициальный комплекс со следуюшими длинами ребер: $N A_{1}^{2}=41, N B_{1}^{2}=34, N A_{2}^{2}=29, N B_{2}^{2}=26$, $A_{1} B_{1}^{2}=25, B_{1} A_{2}^{2}=13, A_{2} B_{2}^{2}=5, B_{2} A_{1}^{2}=17, S A_{1}^{2}=52, S B_{1}^{2}=45, S A_{2}^{2}=40$, $S B_{2}^{2}=37$ (обозначения см. на рис. 3 ). Минимальное (по степени) полиномиальное уравнение для объема, найденное по методу работы [2], имеет следующий вид:

$$
\begin{aligned}
v^{8} & -97600 v^{7}+2150278656 v^{6}-14733766400 v^{5}+28949731124248576 v^{4} \\
& -16429559369328230400 v^{3}+2673932358387945701376 v^{2} \\
& -135342229652751620505600 v+1546362629160356875862016=0,
\end{aligned}
$$

\footnotetext{
${ }^{8}$ Кстати, получаем, что число возможных реализаций данной развертки с числом вершин $n$ и топологического рода $g$ в общем случае не больше, чем $2 N K_{1} K_{2} \ldots K_{e}$, где $2 N$ - степень многочлена для объема, $2 K_{i}, 1 \leqslant i \leqslant e,-$ степени многочленов (9) для малых диагоналей, а $e=3 n-6+6 g$ - число ребер многогранника; учтено, что при известной длине малой диагонали одну грань к другой можно приклеивать двумя способами. Конечно, на самом деле верхняя оценка меньше, так как между малыми диагоналями в звезде каждой вершины есть три связи (см. [7], [8], [15]).
} 


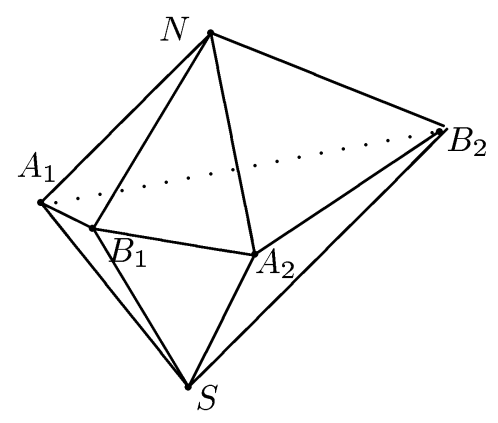

Рис. 3. Модель октаэдра

где $v=36 V^{2}, V$ - объем многогранника. Корнями этого уравнения являются числа $16,64,144,576,1936,7744,17424,69696$, что соответствует восьми возможным значениям квадрата объема $V^{2}=4 / 9,16 / 9,4,16,484 / 9,1936 / 9,484,1936$. Для каждой из трех диагоналей $D_{1}, D_{2}, D_{3}$ октаэдра, которые все являются малыми, составлялось уравнение вида (9), затем из него и из (20) исключалась переменная $V$ и получалось уравнение, в котором коэффициенты зависели только от длин ребер. После нахождения неотрицательных корней $d^{2}$ каждого из этих трех уравнений для каждого из восьми значений $V^{2}$ производился отбор соответствующих значений диагоналей и после этого уж определялись допустимые комбинации значений трех диагоналей, для которых в $\mathbb{R}^{3}$ можно было найти октаэдр, реализующий эти значения длин ребер и диагоналей. Ниже приводится распечатка списка потенциально возможных значений объема и диагоналей октаэдров в $\mathbb{R}^{3}$ с данньми выше длинами ребер, а в конце счета для каждого значения объема даны отобранные значения реально существующих диагоналей:

$$
\begin{array}{rlrl}
V^{2}=4 / 9, \quad d_{1}= & {[2.081694436,4.000000000,31.44166642,31.73100134]} \\
V^{2}=16 / 9, \quad d_{1}= & {[2.021168018,2.175016267,2.312589081,16.00000000,} \\
& 31.23214714,31.77887004] \\
V^{2}=4, & d_{1}= & {[2.035877753,2.304949497,4.000000000,25.52184877,} \\
& 30.98377340,31.61511810] \\
V^{2}=16, \quad d_{1}= & {[2.313393820,2.936605635,16.00000000,29.99931417]} \\
V^{2}=484 / 9, \quad d_{1}= & {[2.060243697,3.360259116,4.000000000,5.018904852,} \\
& 27.37671706,31.57700220] \\
V^{2}=1936 / 9, \quad d_{1}= & {[2.567442580,3.740236092,4.308719174,9.301471323,} \\
& 16.00000000,26.66250012] \\
V^{2}=484, \quad d_{1}= & {[4.000000000,7.485206007,9.084766929,26.47949383]} \\
V^{2}=1936, \quad d_{1}= & {[16.00000000,27.76308932]} \\
V^{2}=4 / 9, \quad d_{2}= & {[3.576012742,3.653258653,4.000000000,10.20814794,} \\
& 39.85885066,40.11735153] \\
V^{2}= & 16 / 9, \quad d_{2}= & {[3.579239880,3.736961331,4.000000000,27.13688493,} \\
& 39.68513202,40.20288543]
\end{array}
$$




$$
\begin{aligned}
& V_{2}=4, \quad d_{2}=[3.610222062,3.852077009,5.677424734,36.00000000, \\
& 39.48078587,40.25796612] \\
& V_{2}=16, \quad d_{2}=[3.863268530,4.400763198,19.74547423,36.00000000, \\
& 38.67277165,40.26348038] \\
& V^{2}=484 / 9, \quad d_{2}=[4.000000000,4.813288766,6.119238093,6.360328867, \\
& 36.56370024,39.71220656] \\
& V^{2}=1936 / 9, \quad d_{2}=[4.000000000,5.191888121,5.836989071,9.835911172 \text {, } \\
& 24.51784495,35.35659901] \\
& V^{2}=484, \quad d_{2}=[5.415779901,9.316632439,11.32390248,36.00000000] \\
& V_{2}=1936, \quad d_{2}=[16.49162805,36.00000000] \\
& V^{2}=4 / 9, \quad d_{3}=[.9747869856,1.000000000,1.054234393,74.68927287, \\
& 120.5563512,121.4151441] \\
& V^{2}=16 / 9, \quad d_{3}=[.9748130474,1.000000000,1.142932772,85.19747307, \\
& 120.0833829,121.8025063] \\
& V^{2}=4, \quad d_{3}=[1.190766164,1.196156004,1.271393891,93.19502451, \\
& \text { 119.5801870, 122.1627160, 1., 1.] } \\
& V^{2}=16, \quad d_{3}=[1.000000000,1.045100811,1.203486490,1.204323642, \\
& \text { 1.925714648, 2.309423273, 3.145288082, 108.7713509, } \\
& 117.8786745,123.0851900] \\
& V^{2}=484 / 9, \quad d_{3}=[1.177526555,1.408032135,1.888971747,2.062233883, \\
& \text { 4.002344336, 5.939546784, } 9.386696355,114.3225064 \text {, } \\
& 121.0000000,124.0951087] \\
& V^{2}=1936 / 9, \quad d_{3}=[1.969558108,2.992830021,4.917251896,5.847359378, \\
& 13.96859475,24.36678384,43.62897578,102.0412689 \text {, } \\
& 121.0000000,124.1867124] \\
& V^{2}=484, \quad d_{3}=[3.315078265,5.724170843,10.32464360,12.69018056, \\
& \text { 41.05804879, 73.51321607, 121., 121.] } \\
& V^{2}=1936, \quad d_{3}=[11.14259787,23.22774529,113.5650136,121.0000000] \\
& V^{2}=4 / 9, \quad d_{1}, d_{2}, d_{3}=[4.000000000,3.99999646,1.00000010] \\
& V^{2}=16 / 9, \quad d_{1}, d_{2}, d_{3}=[16.00000000,4.00000114,1.00000000] \\
& V^{2}=4, \quad d_{1}, d_{2}, d_{3}=[4.000000000,36.00001599,1.00000010] \\
& V^{2}=16, \quad d_{1}, d_{2}, d_{3}=[16.00000000,35.99999696,1.00000000] \\
& V^{2}=484 / 9, \quad d_{1}, d_{2}, d_{3}=[4.000000000,3.99999994,120.9999999] \\
& V^{2}=1936 / 9, \quad d_{1}, d_{2}, d_{3}=[16.00000000,3.99999994,121.0000000] \\
& V^{2}=484, \quad d_{1}, d_{2}, d_{3}=[4.000000000,36.00000009,120.9999999] \\
& V^{2}=1936, \quad d_{1}, d_{2}, d_{3}=[16.00000000,36.00000007,121.0000000]
\end{aligned}
$$

Видим, например, что самое большое значение объема $V=44$ (или $V^{2}=1936$ ) соответствует октаэдру $P$ с диагоналями $D_{1}=4, D_{2}=6, D_{3}=11$, которые 
реализуются для вершин со следуюшими координатами:

$$
A_{1}(-4,0,0), \quad A_{2}(2,0,0), \quad B_{1}(0,-3,0), \quad B_{2}(0,1,0), \quad N(0,0,5), \quad S(0,0,-6),
$$

т.е. с вершинами на осях координат. Остальные семь изометричных $P$ реализаций октаэдра получаются зеркальными отражениями октаэдра $P$ относительно координатных плоскостей $x O y, x O z$ и $y O z$; в частности, самый маленький объем $V=2 / 3$ (или $V^{2}=4 / 9$ ) реализуется для октаэдра (с самопересечениями) с вершинами в точках

$$
A_{1}(4,0,0), \quad A_{2}(2,0,0), \quad B_{1}(0,3,0), \quad B_{2}(0,1,0), \quad N(0,0,5), \quad S(0,0,6) .
$$

Между прочим, реальное существование этих восьми изометричных между собой октаэдров доказывает, что минимально допустимая степень многочлена $Q(V)$ для объема октаэдра действительно равна 8.

3. Теперь мы опишем процедуру нахождения длин других диагоналей многогранника, если уже знаем малые диагонали. Основой для этого является формула для нахождения расстояния между двумя точками с известными расстояниями до трех базовы $x$ точек, не лежаших на одной прямой [5]. Длину ребра $\left\langle p_{i} p_{j}\right\rangle$ обозначим через $l_{i j}$ (см. рис. 4 ).

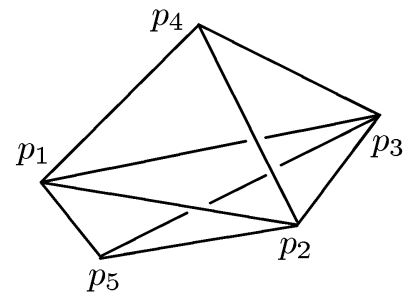

Рис. 4

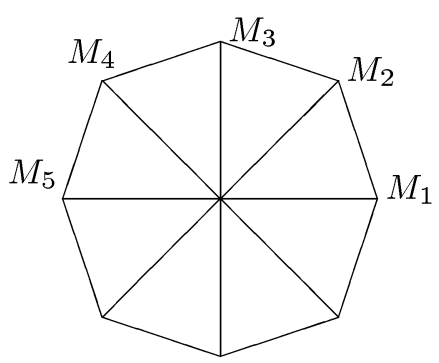

Рис. 5

Далее, пусть $S_{123}$ - площадь треугольника $\left\langle p_{1} p_{2} p_{3}\right\rangle, V_{1234}$ и $V_{1235}$ - объемы соответствующих тетраэдров. Тогда расстояние $d_{45}$ между вершинами $p_{4}$ и $p_{5}$ вычисляется по формуле

$$
d_{45}^{2}=l_{34}^{2}+l_{35}^{2}+\frac{D+36 \epsilon V_{1234} V_{1235}}{S_{123}^{2}},
$$

где

$$
D=\frac{1}{8}\left|\begin{array}{ccc}
2 l_{23}^{2} & l_{12}^{2}-l_{23}^{2}-l_{13}^{2} & l_{25}^{2}-l_{35}^{2}-l_{23}^{2} \\
l_{12}^{2}-l_{23}^{2}-l_{13}^{2} & 2 l_{13}^{2} & l_{35}^{2}+l_{13}^{2}-l_{15}^{2} \\
l_{24}^{2}-l_{23}^{2}-l_{34}^{2} & l_{13}^{2}-l_{34}^{2}-l_{14}^{2} & 0
\end{array}\right|,
$$

a $\epsilon= \pm 1$ в зависимости от того, лежат ли вершины $p_{4}$ и $p_{5}$ по разные или по одну сторону от плоскости треугольника $\left\langle p_{1} p_{2} p_{3}\right\rangle$ (если одна из вершин $p_{4}$ и $p_{5}$ или обе они лежат на плоскости основания, то соответствующие объемы равны нулю и выбор величины $\epsilon$ значения не имеет).

Пусть требуется найти длину диагонали между двумя вершинами, принадлежашими краю одной звезды (рис. 5), скажем, расстояние между вершинами $M_{1}$ и $M_{5}$. 
Расстояния $M_{1} M_{3}, M_{2} M_{4}$ и $M_{3} M_{5}$ известны как длины малых диагоналей. Определяем теперь расстояние между $M_{1}$ и $M_{4}$ по формуле (21) как расстояние между точками с известными расстояниями до вершин базового треугольника $\left\langle O M_{2} M_{3}\right\rangle$, после этого по той же формуле находим расстояние между $M_{1}$ и $M_{5}$ как между точками с известными расстояниями до базовых точек $O, M_{3}$ и $M_{4}$. Очевидно, таким образом мы можем последовательно определить длины всех диагоналей в одной звезде и заодно отбросить неподходящие выборы длин малых диагоналей, так как каждую "не малую" диагональ можно вычислить двумя способами, подходя к рассматриваемой вершине по двум разным направлениям и требуя совпадения результатов вычислений.

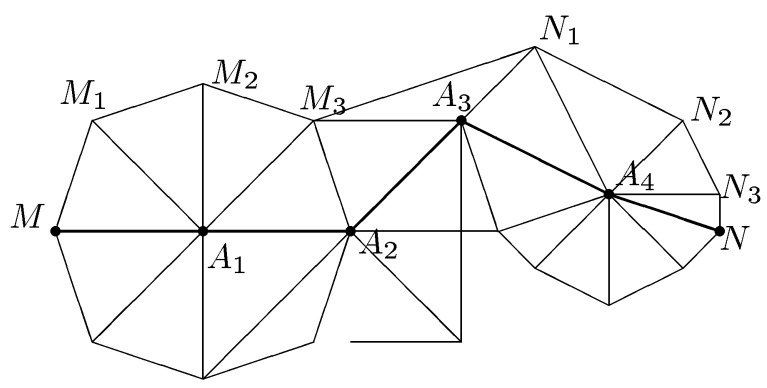

Рис. 6

Пусть теперь диагональ соединяет вершины из разных звезд. Опишем алгоритм вычисления ее длины на примере из рис. 6 для диагонали $M N$. Найдем кратчайший (по числу ребер) путь между точками $M$ и $N$ (на рисунке это ломаная $\left.M A_{1} A_{2} A_{3} A_{4} N\right)$. Расстояния между вершинами внутри одной звезды мы умеем вычислять. Покажем, как найти расстояния от $M$ до вершин звезды $A_{2}$. Звезды вершин $A_{1}$ и $A_{2}$ имеют обшее ребро $\left\langle A_{1} A_{2}\right\rangle$ и обшую грань $\left\langle A_{1} A_{2} M_{3}\right\rangle$, которая является базовым треугольником для определения расстояния между $M$ и $A_{3}$ по формуле (21), так как все расстояния от них до вершин грани $\left\langle A_{1} A_{2} M_{3}\right\rangle$ известны. Нетрудно проверить, что грань $\left\langle A_{2} A_{3} M_{3}\right\rangle$ - базовая для определения расстояния между $M$ и $N_{1}$; после определения этого расстояния грань $\left\langle M_{3} A_{3} N_{1}\right\rangle$ становится базовой для определения расстояния $M A_{4}$. Наконец, известные расстояния от $M$ и $N$ до вершин грани $\left\langle A_{3} A_{4} N_{1}\right\rangle$ позволяет определить расстояние между $M$ и $N$. Конечно, не надо забывать, что на каждом шаге мы находим дв $a$ возможных значения вычисляемых расстояний, поэтому в итоге получаем не точные значения расстояний, а некоторый конечный набор их возможных значений, что и достаточно для конечности алгоритма. Как видим, алгоритм работает в предположении, что в многограннике нет трех вершин, лежаших на одной прямой, иначе какой-нибудь базовый треугольник окажется вырожденным и тогда не удастся применить формулу (21).

Для окончательного решения проблемы изометрической реализации данной развертки можно пойти двумя путями. Если необходимо узнать только существование искомого многогранника, то можно сначала найти расстояния между всеми парами вершин будущего многогранника, а затем воспользоваться известным критерием (см., например, [11]) существования в трехмерном пространстве $n$ точек с данными расстояниями между ними (в силу треугольности граней, знания комбинаторной схемы сушествования вершин достаточно для утверждения сушествова- 
ния ребер и граней). Но если необходимо явно найти многогранник, то для однозначного определения положения вершин достаточно знать их расстояния от четырех вершин, не лежащих на одной плоскости; следовательно, не нужно искать все расстояния, а сначала нужно найти четыре вершины, составляющие невырожденный тетраэдр, искать расстояния от остальных вершин только до этих четырех вершин и для найденных таким образом вершин проверить, определяют ли они ребра именно той длины, которые предписаны данной разверткой.

4. Итак, пока остаются нерешенными лишь случаи, когда имеется достаточно много малых диагоналей, для которых все коэффициенты уравнений вида (9) оказываются равными нулю при соответствующих значениях объема и длин ребер. Но эти случаи бывают только для исключительных значений длин ребер, когда они удовлетворяют довольно большому количеству алгебраических уравнений с целыми коэффищиентами.

Теорема 2 была объявлена ранее в тезисах нескольких конференций, но полное ее доказательство удалось оформить только в июне 2000 г. во время пребывания автора в университете Париж-7. Автор, пользуясь приятной возможностью, благодарит профессора этого университета Гарольда Розенберга за его любезное приглашение.

\section{Список литературы}

1. Александров А. Д. Выпуклые многогранники. М.- Л.: Гостехиздат, 1950.

2. Астрелин A.B., Сабитов И. Х. Минимальный по степени многочлен для определения объема октаэдра по его метрике // УМН. 1992. Т. 50. № 5. С. 245-246.

3. Астрелин А.В., Сабитов И. Х. Канонический многочлен для объема многогранника // УМН. 1999. Т. 54. № 2. С. 165-166.

4. Бураго Ю. Д., Залгаллер В.А. Изометрические кусочно линейные погружения двумерных многообразий с полиэдральной метрикой в $\mathbb{R}^{3} / /$ Алгебра и анализ. 1995. Т. 7. № 3. C. $76-95$.

5. Сабитов И.Х. Алгоритмическая проверка изгибаемости подвесок // Укр. геом. сб. 1987. Т. 30. С. 109-112.

6. Сабитов И. Х. К проблеме об инвариантности объема изгибаемого многогранника // УМН. 1995. Т. 50. № 2. С. 223-224.

7. Сабитов И. Х. Объем многогранника как функция его метрики // Фундам. и прикладн. математика. 1996. Т. 2. №4. С. 1235-1246.

8. Сабитов И. Х. Обобщенная формула Герона-Тарталья и некоторые ее следствия // Матем. сб. 1998. Т. 189. № 10. С. 105-134.

9. Сабитов И. Х. Решение многогранников // Докл. РАН. 2001. Т. 377. № 2. С. 161-164.

10. Сабитов И. Х. Некоторые применения формулы для объема многогранников // Тр. конференции "Геометрия и приложения", посвященной 70-летию В.А. Топоногова. Новосибирск: Изд-во Института математики СО РАН, 2001. С. 183-197.

11. Blumental L. M. Theory and Applications of Distance Geometry. N. Y.: Chelsea, 1970.

12. Gluck H. Almost all simple connected closed sutfaces are rigid // Lec. Notes in Math. 1975. V. 438. P. 225-239.

13. Graver J. Rigidity of triangulated surfaces // Colloquium during the Special Semester on Structural Rigidity. Université de Monréal: Centre de recherches Mathématiques, 1987.

14. Fogelsanger A. The generic rigidity of minimal cycles. Preprint/Cornell University, 1987.

15. Sabitov I. Kh. The Volume as a Metric Invariant of Polyhedra // Discrete and Computat. Geometry. 1998. V. 20. № 4. P. 405-425.

16. Sabitov I. Kh. On some recent results in the metric theory of polyhedra // Supplemento ai Rendiconti del Circolo Mat. di Palermo. Serie II. 2000. № 65. P. 167-177.

17. Whiteley $W$. Infinitesimal rigid polyhedra III: triangulated tori. Preprint/ Champlain Regional College, St. Lambert, Quebec J4P 3B8, 1985. 